SUBJECT AREAS:

PRE-CLINICAL STUDIES

CELL DEATH IN THE NERVOUS

SYSTEM

Received

8 October 2013

Accepted

16 December 2013

Published

13 January 2014

Correspondence and requests for materials should be addressed to

L.M. (Michael.

Luchtmann@med.

ovgu.de) \section{resonance angiography in brain death}

\author{
Luchtmann M. ${ }^{\text {, }}$ Beuing O. ${ }^{2}$, Skalej M. ${ }^{2}$, Kohl J. ${ }^{\text {, , Serowy S. }}{ }^{2}$, Bernarding J. ${ }^{3}$ \& Firsching R. ${ }^{1}$
}

${ }^{1}$ Department of Neurosurgery, Otto-von-Guericke-University Magdeburg, ${ }^{2}$ Institute of Neuroradiology, Otto-von-Guericke-
University Magdeburg, ${ }^{3}$ Institute of Biometry and Medical Informatics, Otto-von-Guericke-University Magdeburg.

Confirmatory tests for the diagnosis of brain death in addition to clinical findings may shorten observation time required in some countries and may add certainty to the diagnosis under specific circumstances. The practicability of Gadolinium-enhanced magnetic resonance angiography to confirm cerebral circulatory arrest was assessed after the diagnosis of brain death in 15 patients using a 1.5 Tesla MRI scanner. In all 15 patients extracranial blood flow distal to the external carotid arteries was undisturbed. In 14 patients no contrast medium was noted within intracerebral vessels above the proximal level of the intracerebral arteries. In one patient more distal segments of the anterior and middle cerebral arteries (A3 and M3) were filled with contrast medium. Gadolinium-enhanced MRA may be considered conclusive evidence of cerebral circulatory arrest, when major intracranial vessels fail to fill with contrast medium while extracranial vessels show normal blood flow.

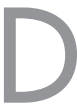

eath is the irreversible loss of all functions. The diagnosis of brain death in most countries is based on the evidence of the loss of brain function and confirmation of the irreversibility of that loss of function ${ }^{1-4}$. The clinical criteria giving evidence of the loss of brain function (coma, cranial nerve areflexia and apnea) are universally accepted. "Most physicians recognize that the diagnosis of death based upon clinical judgement alone is subject to human error"s. The evidence for the irreversibility of loss of function, a waiting period and/or a confirmatory test, is viewed variably and reflects different concepts of safety ${ }^{6,7}$.

Two main groups of confirmatory tests focus either on the demonstration of the cessation of cerebral blood flow or the absence of cerebral bioelectrical brain activity. Conventional angiography and electroencephalography (EEG) have frequently been recommended ${ }^{4}$. As the complete arrest of cerebral blood flow will result in a global brain infarction after a short interval ${ }^{8}$ Bernat ${ }^{1}$ considered the registration of the absence of intracranial blood flow the most reliable finding to demonstrate an irreversible loss of all brain functions. Identification of cerebral circulatory arrest has been reported using a number of techniques: computed tomography (CT) angiography, four-vessel angiography, cerebral digital subtraction angiography, intravenous radionuclide angiography, single photon emission computed tomography (SPECT), and transcranial Doppler ultrasonography $(\mathrm{TCD})^{9-14}$. All these techniques have their limitations and their availability is variable. While TCD depends on the expertise of the investigator and requires a high degree of experience, CT angiography (CTA) involves contrast media with a possibly harmful side effect on the donor or the transplantable organs.

We consider contrast-enhanced MR-angiography (MRA) particularly suitable to identify cerebral circulatory arrest. Subsequently we report our MRA findings, which we obtained in 15 patients after brain death had been diagnosed.

\section{Results}

The clinical data and the MR findings of all brain dead patients are presented in Table 1. Common MRI findings were diffuse gyral swelling, parenchymal hemorrhage; diffuse cerebral white matter injury and tonsilar herniation. In 14 of 15 patients the Gadolinium-enhanced MRA showed no evidence of arterial blood flow in the intracranial circulation above the level of the proximal A2-segment of the anterior cerebral artery (ACA) and the M1-segment of the middle cerebral artery (MCA) as depicted in Figure 1. One patient exhibited some extremely slow cerebral blood flow of contrast medium into the A3 and M3 segments after the diagnosis of brain death (Figure 2).

\section{Discussion}

Confirmatory tests for the diagnosis of $\mathrm{BD}$ in addition to the clinical findings of coma and apnoeic cranial nerve areflexia are not required in many countries ${ }^{4}$. It may be mandatory in some countries under specific clinical 


\begin{tabular}{|c|c|c|c|}
\hline 1 & $\mathrm{ICH}$ & $\begin{array}{l}\text { Intraventricular hemorrhage, parenchymal hemorrhage, diffuse gyral swelling, } \\
\text { tonsillar herniation }\end{array}$ & No flow \\
\hline 2 & SAH & Diffuse gyral swelling, tonsillar herniation & No flow \\
\hline 3 & SAH & Diffuse gyral swelling, tonsillar herniation & No flow \\
\hline 4 & $\mathrm{ICH}$ & $\begin{array}{l}\text { Intraventricular hemorrhage, parenchymal hemorrhage, diffuse gyral swelling, } \\
\text { tonsillar herniation }\end{array}$ & No flow \\
\hline 6 & Trauma & Diffuse cerebral white matter injury, diffuse gyral swelling, tonsillar herniation & No flow \\
\hline 7 & Trauma & Intraventricular hemorrhage, diffuse gyral swelling, tonsillar herniation & No flow \\
\hline 8 & $\mathrm{ICH}$ & Parenchymal hemorrhage, diffuse gyral swelling, tonsillar herniation & No flow \\
\hline 9 & $\mathrm{ICH}$ & $\begin{array}{l}\text { Diffuse cerebral white matter injury, parenchymal hemorrhage, diffuse gyral } \\
\text { swelling, tonsillar herniation }\end{array}$ & No flow \\
\hline 10 & Trauma & Diffuse gyral swelling, tonsillar herniation & $\begin{array}{l}\text { Extremely slow cerebral } \\
\text { blood flow }\end{array}$ \\
\hline 14 & Trauma & Diffuse gyral swelling, tonsilar herniation & No flow \\
\hline 15 & Trauma & Diffuse cerebral white matter injury, diffuse gyral swelling, tonsilar herniation & No flow \\
\hline
\end{tabular}

conditions ${ }^{6}$. Confirmatory tests are used in cases of unreliable clinical examinations, to shorten the time of observation, and in some countries in primary brain lesions of the posterior fossa. The treatment with CNS depressant medication (e.g. barbiturates) is the most frequent reason for the delay of the diagnosis of brain death based on clinical criteria. As long as CNS depressant drugs have not been eliminated to a neglectable level or until other confounding factors such as metabolic or endocrine disorders have not been excluded, brain death cannot be diagnosed on clinical criteria alone. A delay of the diagnosis of brain death has been linked to an increased risk of organ failure in transplant recipients ${ }^{15}$ as timing has proved to be an important factor for successful transplantation. In order to preserve the integrity of the donor organs and to decrease the rate of transplant failures some authors therefore call for a regular use of reliable confirmatory tests to shorten the time needed to diagnose brain death ${ }^{16}$. Confirmatory tests may be divided into two groups: in those that demonstrate the cessation of cerebral blood flow (CBF) and those, which prove the absence of bioelectrical activity. In the countries in which confirmatory tests are recommended or eventually even required, the most often used ancillary test is electroencephalography (EEG), which has been reported to have a $90 \%$ sensitivity $^{2}$. Other accepted electrophysiological tests rely on the evidence of the loss of evoked potentials ${ }^{17}$. A reliable confirmatory test would be most helpful particularly in cases when cranial nerve areflexia cannot be verified because of lesions to the midface and the eyes or in concomitant drug intoxication.

In recent years great efforts have been made to develop new confirmatory tests for the reliable diagnosis of brain death. Magnetic resonance imaging (MRI) is believed to offer several sophisticated techniques that may improve the diagnostics of BD. Aichner et al. ${ }^{18}$ observed uniform patterns of diffuse brain swelling and tonsilar herniation in patients with clinical signs of brain death. Kumada et al. ${ }^{19}$ found decreased ADC values up to $40 \%$ as compared to a healthy control group emphasizing that DWI and ADC mapping show areas that correspond to edema of cytotoxic nature and ischemic tissue. Several other authors confirmed these results ${ }^{20-23}$. In a recent study we evaluated the reliability of diffusion weighted imaging in the diagnosis of brain death. We concluded that the unpredictable effects of pseudonormalization and possible susceptibility artifacts due to micro hemorrhages preclude a leading role of DWI in the diagnosis of brain death ${ }^{22}$.

It is widely accepted that the cessation of cerebral blood flow for about 30 minutes results in a global brain infarction and subsequently in brain death ${ }^{8}$. Hence, Bernat ${ }^{1}$ argued that the proof of a complete cerebral circulatory arrest is the most reliable confirmation of an irreversible loss of all brain functions. The precise pathophysiology of the cessation of cerebral blood flow may still be discussed, but several mechanisms have been proposed to explain the arrest of brain circulation in BD. A massively increased intracerebral pressure (ICP) leads to an increased vascular resistance of the brain. When the ICP exceeds the systolic blood pressure, the brain cannot be perfused. As the tolerance of brain tissue to withdrawal of oxygen is very short, thus irreversible destruction of brain tissue will ensue within minutes, leading to further swelling of the brain. Undoubtedly, a total cerebral circulatory arrest documents the "death of all intracranial neurons" ${ }^{\prime 1,24}$. Various techniques have been used to demonstrate this arrest: four-vessel angiography ${ }^{12}$, cerebral intravenous digital subtraction angiography ${ }^{9}$, radionuclide angiography ${ }^{10}$, single photon emission tomography ${ }^{25}$, MRI angiography ${ }^{26-29}, \mathrm{CT}$ angiography ${ }^{13}$, and a few more ${ }^{2}$. Transcranial Doppler sonography (TCD) is a non-invasive technique and is independent from any contrast media. It is costeffective and additionally fast and easy to use on the intensive care unit $(\mathrm{ICU})^{30}$. Some authors have investigated the reliability of MR angiography ${ }^{26,27,29}$. Ishii et al. ${ }^{26}$ suggested first that absence of the cerebral blood flow above the supraclinoid portions of ICA and their intracranial branches on MR angiograms is specific to brain death. Karantanas et $\mathrm{al}^{27}$ and Sohn et al. ${ }^{29}$ confirmed these findings using sophisticated time-of-flight (TOF) imaging techniques. TOF imaging has been increasingly accepted as a technique for the examination of the intracranial circulation. The advantages of time-of-flight imaging include high spatial resolution, relative short imaging time, high signal-to-noise ratio and the independence from any contrast agents $^{31}$. It is based on the flow-related signal enhancement of the blood entering into the volume-of-interest ${ }^{32}$. TOF imaging, however, underlies some technical limitations and potential pitfalls. Large intracranial hematomas excite high signal intensities leading to an extremely difficult and unreliable interpretation of the intracranial circulation $^{33}$. The dependence on adequate flow enhancement imposes certain constraints on time-of-flight imaging. Thus, slowed circulation causes dephasing and saturation leading to signal losses. Furthermore, when vessels run parallel to the imaging slice orientation, the signal may become saturated with a subsequently undetectable blood flow, even when the blood flow is not severely impaired ${ }^{31}$.

As we are not aware of any preceding study examining the diagnostic value of gadolinium-enhanced MR angiography (MRA) for the detection of brain death we examined its practicability to confirm 

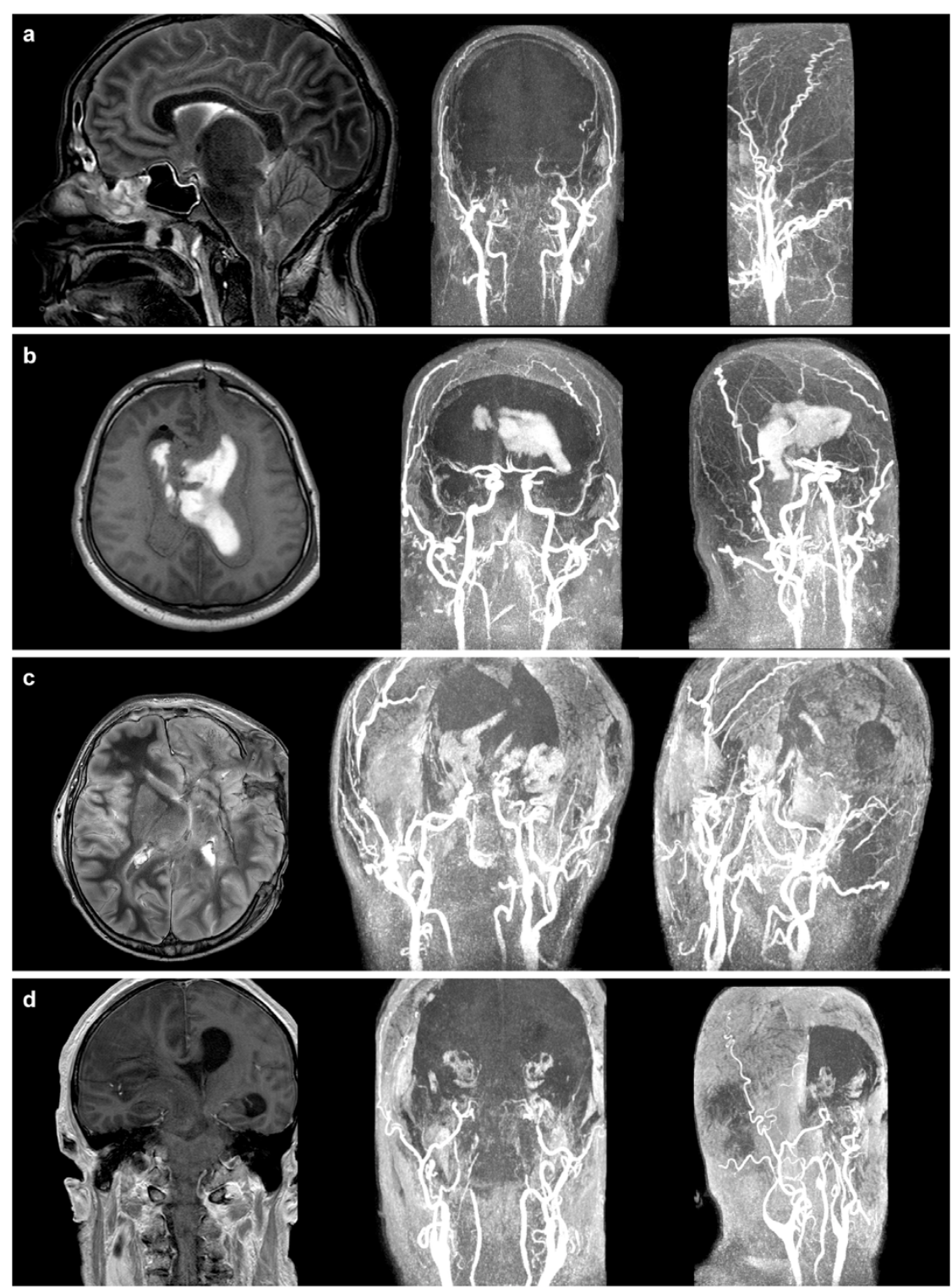

Figure $1 \mid$ MRI/MRA of two patients diagnosed with clinical brain death. a) The T2-weighted sagittal image shows tonsilar herniation due to global brain edema as one of the common findings in brain dead patients. The gadolinium-enhanced MR angiography showed no evidence for arterial blood flow in the intracranial circulation. b) The T1-weighted axial image shows diffuse gyral swelling, intraventricular hemorrhage and a fracture of the anterior skull resulting from severe traumatic head injury. The MRA showed no intracranial blood above the level of the proximal A2-segment of the ACA and the M1segment of the MCA. c) The T2-weighted axial image depicts the situation after left-sided unilateral decompressive craniectomy showing a midline shift after massive hemispheric infarction due to vasospasm resulting from a subarachnoid hemorrhage. The MRA showed no evidence for cerebral blood flow while extracranial vessels exhibit normal circulation. d) The coronal T1-weighted image shows decompression after a hemorrhagic stroke affecting the right hemisphere. The MRA confirms the absence intracerebral arterial blood flow.

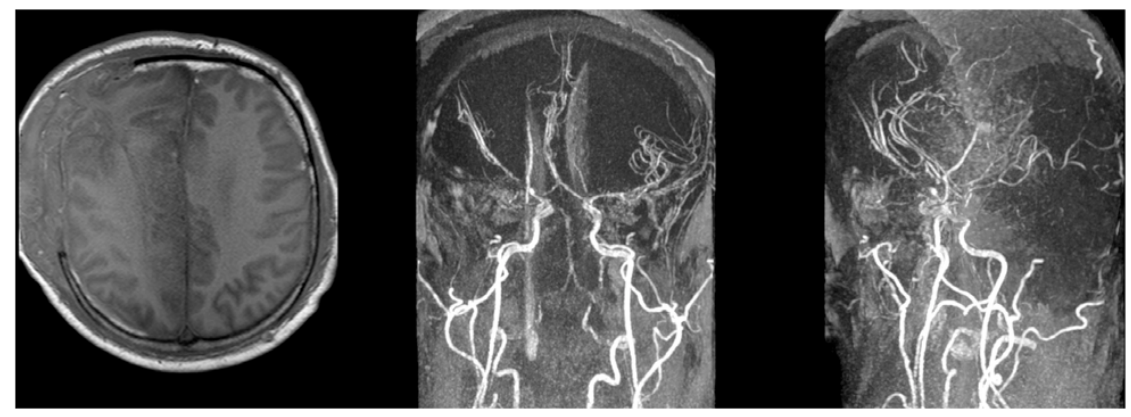

Figure $2 \mid$ MRI/MRA of a patient diagnosed with clinically diagnosed brain death. The T1-weigthed axial image shows the situation after right-sided unilateral decompressive craniectomy following severe traumatic brain injury. MRA shows contrast medium in A3 and M3 segments. Compared with the contrast medium within the extracranial vessels an extremely slow intracerebral circulation is obvious. 
cerebral circulatory arrest. In 14 patients the MRA showed no intracranial contrast signal media above the level of the proximal A2segment of the ACA and the M1-segment of the MCA indicating an irreversible loss of all brain functions (Figure 1). These results are consistent with the findings of several other authors who investigated the intracranial circulation using TOF-based MR angiography ${ }^{26,27,29}$. No blood flow above the level of the supraclinoid portions of the ICA was found. One brain dead patient presented with contrast medium in the A3 and M3 segments (Figure 2). Such findings are not unusual, as "total cessation of circulation is less common than reduced cerebral blood flow in the early phases of brain death" . Flower and Patel ${ }^{34}$ observed some markedly reduced residual intracranial arterial flow in 6 patients who were brain dead using radionuclide angiography. Shah et al. $^{35}$ evaluated the reliability of cerebral perfusion scintigraphy (CPS) in the diagnosis of brain death. They reported the case of a patient who suffered brain death as a result of a ruptured giant aneurysm and following subarachnoid hemorrhage. A partially persistent intracranial perfusion was detected by using CPS. Several authors presented persistent but reduced CBF in patients after diagnosis of brain death using various imaging techniques ${ }^{36,37}$ in up to $2-$ $3 \%{ }^{38}$. Decreased intracranial pressure e.g. after decompressive craniectomy, extensive decompressing fractures or ventricular shunts may lead to preserved but markedly decreased cerebral blood flow or some reperfusion after the onset of complete and irreversible loss of brain function $^{38}$. In the present study patient \#10 exhibited a markedly reduced intracranial circulation after unilateral decompressive craniectomy and subsequent diagnosis of brain death (Figure 2). These observations from angiography have led to the concept that the documentation of cerebral circulatory arrest is conclusive evidence of brain death. Some residual blood flow is compatible with brain death but less conclusive than large vessels failing to fill with contrast medium while extracranial vessels exhibit normal circula$\operatorname{tion}^{39}$. In the case of residual cerebral blood the diagnosis of brain death cannot be ruled out and diagnosis should be based either on the duration of observation time or other confirmatory tests.

An ideal confirmatory test for the diagnosis of brain death is safe, extremely sensitive, reliable, and widely available. None of the existing ancillary tests fulfill all these criteria. Validities and sensitivities of $90 \%$ and more were reported for the widely accepted ancillary tests that confirm brain death by either demonstrating the absence of cerebral bioelectrical activity or cerebral circulation ${ }^{2,14}$. Bedside examinations are more practicable on the intensive care unit (ICU) and MRI of ventilated patients is quite a burden in terms of time and personnel required. All confirmatory tests, however, have their limitations. TCD is investigator-dependent and requires a high degree of experience. Four-vessel and CT angiography are based on contrast media with a potentially harmful impact on the possibly alive patients and on possible donor organs. Direct arterial angiography is hardly performed in Germany because of legal concerns, since adverse effects of iodine contrast media and arterial infarction are a rare but realistic risk ${ }^{40}$. EEG is useless in hypothermia, CNS depressants and hypotonic conditions ${ }^{2}$. Angiographic methods that demonstrate the absence of cerebral blood flow are independent from confounders like drug-intoxication or metabolic disturbances that preclude the $\mathrm{BD}$ diagnosis on clinical criteria solely.

The presented preliminary results of gadolinium-enhanced MRA likewise show highest sensitivity and validity. To date, now study compared the validity and reliability between contrast-enhanced (CE-MRA) and non-contrast-enhanced magnetic resonance angiography (NCA-MRA) and its practicability in the diagnosis of BD. Both methods showed sensitivities and specificities up to $100 \%$. The substantial differences, however, are based on the methodical issues ${ }^{26,27,29}$. Thus, CE-MRA relies on the paramagnetic properties of the applied contrast agents like Gadolinium (Gd) that shorten the T1 relaxation time. In contrast, NCE-MRA techniques, such as TOF, are based on the suppression of the background signal by slice-selective

\begin{tabular}{lcc}
\multicolumn{3}{l}{ Table 2 | Imaging parameters of the used pulse sequences } \\
Sequence & TR/TE/IT & Slice thickness \\
\hline Sagittal T2 TSE & $10,000 / 120 \mathrm{~ms}$ & $3 \mathrm{~mm}$ \\
Coronal T2 TSE & $11,000 / 120 \mathrm{~ms}$ & $3 \mathrm{~mm}$ \\
Axial T2 TSE & $4.000 / 120 \mathrm{~ms}$ & $5 \mathrm{~mm}$ \\
Axial T1 SE & $550 / 13 \mathrm{~ms}$ & $5 \mathrm{~mm}$ \\
Axial T2* GRE & $850 / 23 \mathrm{~ms}$ & $5 \mathrm{~mm}$ \\
Coronal T2 FLAIR & $6000 / 120 / 2000$ & $5 \mathrm{~mm}$ \\
CE GRE MRA & $4.9 / 1.7$ & $1.2 \mathrm{~mm}$ iso voxel
\end{tabular}

Note: TR - repetition time, TE - echo time, IT - inversion time, TSE - turbo spin echo, GRE - gradient echo, FLAIR - fluid attenuated inversion recovery, CE contrastenhanced.

radiofrequency pulses that saturate the signal from the stationary tissue. NCE-MRA techniques fully refrain from the use of contrast agents. Advantages of CE-MRA relative to NCE-MRA techniques include considerably shorter acquisition times, improved anatomical coverage, and decreased susceptibility artifacts caused by hemorrhages or other pathological conditions like increased intracranial pressure and reduced intracranial blood flow ${ }^{41}$. The major drawback of CE-MRA is the dependence on contrast agents such as Gd that is known to induce nephrogenic systemic fibrosis (NSF); a very rare syndrome however, which appears to occur exclusively in patients with renal impairment. Severe, life-threatening anaphylactoid or nonallergic anaphylactic reactions are exceedingly rare with a likelihood of about $1: 1,000,000$ patients $^{42}$. No case of NSF has been reported in patients with unimpaired renal function as of yet ${ }^{43}$. The effect of contrast media on organ transplants is unknown as of yet. The unwanted side effects of invasive arterial angiography with iodine contrast media have raised generally accepted legal concerns. The likelihood of severe unwanted side effects of iodine contrast media is $1: 170,000^{44}$. Thus, MR angiography with Gd appears to pose a tenfold lower risk to the patient than CT angiography with iodine contrast media.

We concluded that contrast-enhanced MRA techniques may be useful to confirm brain death and that its inherent risk is acceptably low. A comparison of CE- and NCE-MRA in future studies may be interesting.

\section{Methods}

The present study was approved by the Local Ethics Committee of the University of Magdeburg in compliance with national legislation and the Code of Ethical Principles for Medical Research Involving Human Subjects of the World Medical Association (Declaration of Helsinki).

Patients. Gadolinium-enhanced MRA of the brain was prospectively obtained in fifteen patients ( 13 males and 2 females; mean age $58.20 \pm 19.6$ years; age ranged between 20 and 80 years). The primary underlying brain lesion was caused by: traumatic brain injury $(n=6)$, intracerebral hemorrhage $(n=7)$, and subarachnoid hemorrhage $(n=2)$.

Brain death was diagnosed according to the guidelines of the Bundesärztekammer [Richtlinien zur Feststellung des Hirntodes, Deutsches Ärzteblatt 95, Heft 30, 24.07.1998]. Clinical tests included the confirmation of coma, cranial nerve areflexia, apnea and the loss of cerebral bioelectrical activity using EEG for at least 30 minutes as a sign of irreversible bioelectrical silence. The presence of a brain lesion and exclusion of other factors that can cause coma was a prerequisite (e.g. CNS-depressant drugs, hypothermia, imbalance of electrolytes, metabolic or endocrine disturbances).

Imaging. All patients were examined within the first 24 hours after the clinical diagnosis of brain death. MR imaging was conducted on a 1.5 Tesla Intera scanner (Philips Medical Systems, Best, Netherlands) using a 16-channel head coil. Details of the MRA protocol are listed in Table 2.

The MR angiography was visualized by a maximum intensity projection (MIP).

Data analysis. Image data of all observed brain dead patients were pseudonymized and transferred to a separate workstation running Osirix [Version 5.5.2, http://www. osirix-viewer.com/]. The MRA findings were evaluated by neuroradiologists.

1. Bernat, J. L. On irreversibility as a prerequisite for brain death determination. $A d v$ Exp Med Biol 550, 161-167 (2004). 
2. Machado, C. Diagnosis of brain death. Neurol Int 2, e2, doi:10.4081/ni.2010.e2 (2010).

3. Stevens, R. D. \& Bhardwaj, A. Approach to the comatose patient. Crit Care Med 34, 31-41 (2006).

4. Wijdicks, E. F., Varelas, P. N., Gronseth, G. S. \& Greer, D. M. Evidence-based guideline update: determining brain death in adults: report of the Quality Standards Subcommittee of the American Academy of Neurology. Neurology 74, 1911-1918, doi:10.1212/WNL.0b013e3181e242a8 (2010).

5. Walker, A. E. Cerebral death. 3rd edn, 95 (Urban \& Schwarzenberg, 1985).

6. Haupt, W. F. \& Rudolf, J. European brain death codes: a comparison of national guidelines. J Neurol 246, 432-437 (1999).

7. Wijdicks, E. F. Brain death worldwide: accepted fact but no global consensus in diagnostic criteria. Neurology 58, 20-25 (2002).

8. Ingvar, D. H. Brain death-total brain infarction. Acta Anaesthesiol Scand Suppl 45, 129-140 (1971).

9. Albertini, A., Schonfeld, S., Hiatt, M. \& Hegyi, T. Digital subtraction angiographya new approach to brain death determination in the newborn. Pediatr Radiol 23, 195-197 (1993).

10. Braunstein, P., Korein, J., Kricheff, II. \& Lieberman, A. Evaluation of the critical deficit of cerebral circulation using radioactive tracers (bolus technique). Ann NY Acad Sci 315, 143-167 (1978).

11. Combes, J. C., Chomel, A., Ricolfi, F., d'Athis, P. \& Freysz, M. Reliability of computed tomographic angiography in the diagnosis of brain death. Transplant Proc 39, 16-20, doi:10.1016/j.transproceed.2006.10.204 (2007).

12. Munari, M. et al. Confirmatory tests in the diagnosis of brain death: comparison between SPECT and contrast angiography. Crit Care Med 33, 2068-2073 (2005).

13. Qureshi, A. I., Kirmani, J. F., Xavier, A. R. \& Siddiqui, A. M. Computed tomographic angiography for diagnosis of brain death. Neurology 62, 652-653 (2004).

14. Welschehold, S. et al. Technical aids in the diagnosis of brain death: a comparison of SEP, AEP, EEG, TCD and CT angiography. Dtsch Arztebl Int 109, 624-630, doi:10.3238/arztebl.2012.0624 (2012).

15. Ramjug, S., Hussain, N. \& Yonan, N. Prolonged time between donor brain death and organ retrieval results in an increased risk of mortality in cardiac transplant recipients. Interact Cardiovasc Thorac Surg 12, 938-942, doi:10.1510/ icvts.2010.252809 (2011)

16. Lopez-Navidad, A. et al. Early diagnosis of brain death in patients treated with central nervous system depressant drugs. Transplantation 70, 131-135 (2000).

17. Firsching, R. \& Frowein, R. A. in Head Injuries Vol. 17 Advances in Neurosurgery (eds ReinholdA Frowein, Mario Brock, \& Margareta Klinger) Ch. 48, 275-280 (Springer Berlin Heidelberg, 1989).

18. Aichner, F. et al. Magnetic resonance: a noninvasive approach to metabolism, circulation, and morphology in human brain death. Ann Neurol 32, 507-511, doi:10.1002/ana.410320405 (1992).

19. Kumada, K. et al. Diffusion-weighted imaging of brain death: study of apparent diffusion coefficient. No To Shinkei 53, 1027-1031 (2001).

20. Lovblad, K. O. \& Bassetti, C. Diffusion-weighted magnetic resonance imaging in brain death. Stroke 31, 539-542 (2000).

21. Nakahara, M., Ericson, K. \& Bellander, B. M. Diffusion-weighted MR and apparent diffusion coefficient in the evaluation of severe brain injury. Acta Radiol 42, 365-369 (2001).

22. Luchtmann, M. et al. Controversies of diffusion weighted imaging in the diagnosis of brain death. J Neuroimaging 23, 463-468 (2013).

23. Selcuk, H. et al. Diffusion-weighted imaging findings in brain death. Neuroradiology 54, 547-554, doi:10.1007/s00234-011-0912-9 (2012).

24. Pallis, C. Whole-brain death reconsidered-physiological facts and philosophy. J Med Ethics 9, 32-37 (1983).

25. Facco, E. et al. $99 \mathrm{mTc}-\mathrm{HMPAO}$ SPECT in the diagnosis of brain death. Intensive Care Med 24, 911-917 (1998).

26. Ishii, K. et al. Brain death: MR and MR angiography. AJNR Am J Neuroradiol 17, 731-735 (1996).

27. Karantanas, A. H., Hadjigeorgiou, G. M., Paterakis, K., Sfiras, D. \& Komnos, A. Contribution of MRI and MR angiography in early diagnosis of brain death. Eur Radiol 12, 2710-2716, doi:10.1007/s00330-002-1336-z (2002).

28. Matsumura, A. et al. Magnetic resonance imaging of brain death. Neurol Med Chir (Tokyo) 36, 166-171 (1996).
29. Sohn, C. H. et al. Imaging findings of brain death on 3-tesla MRI. Korean J Radiol 13, 541-549, doi:10.3348/kjr.2012.13.5.541 (2012).

30. Conti, A. et al. Transcranial Doppler ultrasonography in the assessment of cerebral circulation arrest: improving sensitivity by transcervical and transorbital carotid insonation and serial examinations. Neurocrit Care 10, 326-335, doi:10.1007/s12028-009-9199-7 (2009).

31. Lohan, D. G. S., R. Nael, K., Krishnam, M. \& Finn, J. P. Contrast-enhanced MRA versus nonenhanced MRA: Pros and cons. Applied Radiology 36, 3-15 (2007).

32. Nishimura, D. G. Time-of-flight MR angiography. Magn Reson Med 14, 194-201 (1990).

33. Wilcock, D. J., Jaspan, T. \& Worthington, B. S. Problems and pitfalls of 3-D TOF magnetic resonance angiography of the intracranial circulation. Clin Radiol 50, 526-532 (1995).

34. Flowers, W. M. \& Patel, B. R. Radionuclide angiography as a confirmatory test for brain death: A review of 229 studies in 219 patients. South Med J 90, 1091-1096 (1997).

35. Shah, R., Miron, S. \& Sodee, D. B. Visualization of cerebral aneurysm on Tc-99m HMPAO brain perfusion scintigram for brain death. Clin Nucl Med 19, 457-458 (1994).

36. Nau, R. et al. Results of four technical investigations in fifty clinically brain dead patients. Intensive Care Med 18, 82-88 (1992).

37. Rosenklint, A. \& Jorgensen, P. B. Evaluation of angiographic methods in the diagnosis of brain death. Correlation with local and systemic arterial pressure and intracranial pressure. Neuroradiology 7, 215-219 (1974).

38. Flowers, W. M., Jr. \& Patel, B. R. Persistence of cerebral blood flow after brain death. South Med J 93, 364-370 (2000).

39. Vlahovitch, B. F., P. Kuhner, A., Stopak, B., Allais, B. \& Gros, C. Arrêt circulatoire intracranien dans la mort du cerveau: Angiographie avec injection sous pression. Acta Radiologica 13, 334-349 (1972).

40. König, B. Todesbegriff, Todesdiagnostik und Strafrecht. Kieler Schriften zum Strafrecht. 1st edn, Vol. 9 (Alfred Metzner Verlag, 1989).

41. Hartung, M. P., Grist, T. M. \& Francois, C. J. Magnetic resonance angiography: current status and future directions. J Cardiovasc Magn Reson 13, 19, doi:10.1186/ 1532-429X-13-19 (2011).

42. Prince, M. R., Zhang, H., Zou, Z., Staron, R. B. \& Brill, P. W. Incidence of immediate gadolinium contrast media reactions. Am J Roentgenol 196, W138-143, doi:10.2214/AJR.10.4885 (2011).

43. Wang, Y. et al. Incidence of nephrogenic systemic fibrosis after adoption of restrictive gadolinium-based contrast agent guidelines. Radiology 260, 105-111, doi:10.1148/radiol.11102340 (2011).

44. Morcos, S. K. \& Thomsen, H. S. Adverse reactions to iodinated contrast media. Eur Radiol 11, 1267-1275 (2001).

\section{Author contributions}

M.L. prepared the manuscript including all figures/tables and was responsible for the clinical part of the data collection and analysis. O.B. and M.S. were responsible for the technical part of the data collection. J.K. was responsible for the clinical part of the data collection. S.S. analysed the collected data especially the maximum intensity projections (MIP) of the MRA. J.B. prepared parts of the manuscript and analyzed the collected data R.F. designed the study and was responsible for manuscript preparation and data analysis. All authors reviewed the manuscript.

\section{Additional information}

Competing financial interests: The authors declare no competing financial interests.

How to cite this article: L.M. et al. Gadolinium-enhanced magnetic resonance angiography in brain death. Sci. Rep. 4, 3659; DOI:10.1038/srep03659 (2014).

cc)(1) $\odot$ This work is licensed under a Creative Commons Attribution-

(c) ${ }_{\mathrm{BY}} \mathrm{NC}$ ND NonCommercial-NoDerivs 3.0 Unported license. To view a copy of this license, visit http://creativecommons.org/licenses/by-nc-nd/3.0 